\title{
Video Article \\ A Procedure for Studying the Footshock-Induced Reinstatement of Cocaine Seeking in Laboratory Rats
}

\author{
David A. Kupferschmidt ${ }^{1}$, Zenya J. Brown ${ }^{1}$, Suzanne Erb ${ }^{1}$ \\ ${ }^{1}$ Psychology, University of Toronto Scarborough \\ Correspondence to: Suzanne Erb at erb@utsc.utoronto.ca \\ URL: https://www.jove.com/video/2265 \\ DOI: doi:10.3791/2265
}

Keywords: Neuroscience, Issue 47, Relapse, Reinstatement, Cocaine, Rat, Footshock, Stress, Intravenous, Self-administration, Operant Conditioning

Date Published: 1/6/2011

Citation: Kupferschmidt, D.A., Brown, Z.J., Erb, S. A Procedure for Studying the Footshock-Induced Reinstatement of Cocaine Seeking in Laboratory Rats. J. Vis. Exp. (47), e2265, doi:10.3791/2265 (2011).

\section{Abstract}

The most insidious aspect of drug addiction is the high propensity for relapse. Animal models of relapse, known as reinstatement procedures, have been used extensively to study the neurobiology and phenomenology of relapse to drug use. Although procedural variations have emerged over the past several decades, the most conventional reinstatement procedures are based on the drug self-administration (SA) model. In this model, an animal is trained to perform an operant response to obtain drug. Subsequently, the behavior is extinguished by withholding responsecontingent reinforcement. Reinstatement of drug seeking is then triggered by a discrete event, such as an injection of the training drug, reexposure to drug-associated cues, or exposure to a stressor ${ }^{1}$.

Reinstatement procedures were originally developed to study the ability of acute non-contingent exposure to the training drug to reinstate drug seeking in rats and monkeys ${ }^{1,2}$. Reinstatement procedures have since been modified to study the role of environmental stimuli, including drugassociated cues and exposure to various forms of stress, in relapse to drug seeking ${ }^{1,3,4}$.

Over the past 15 years, a major focus of the reinstatement literature has been on the role of stress in drug relapse. One of the most commonly used forms of stress for studying this relationship is acute exposures to mild, intermittent, electric footshocks. The ability of footshock stress to induce reinstatement of drug seeking was originally demonstrated by Shaham and colleagues (1995) in rats with a history of intravenous heroin $S A^{5}$. Subsequently, the effect was generalized to rats with histories of intravenous cocaine, methamphetamine, and nicotine $\mathrm{SA}$, as well as oral ethanol SA ${ }^{3,6}$.

Although footshock-induced reinstatement of drug seeking can be achieved reliably and robustly, it is an effect that tends to be sensitive to certain parametrical variables. These include the arrangement of extinction and reinstatement test sessions, the intensity and duration of footshock stress, and the presence of drug-associated cues during extinction and testing for reinstatement. Here we present a protocol for footshock-induced reinstatement of cocaine seeking that we have used with consistent success to study the relationship between stress and cocaine seeking.

\section{Video Link}

The video component of this article can be found at https://www.jove.com/video/2265/

\section{Protocol}

\section{Animal Housing and Acclimatization Procedures}

1. Male Long Evans rats, weighing 300-325 $\mathrm{g}$ at the time of arrival in the vivarium, are housed individually in standard plastic cages containing recycled paper pellet bedding, with wire grid lids to accommodate water bottles and food.

2. Rats are maintained on a reverse light-dark schedule (lights on 19:00 to 07:00), in a temperature- and humidity-controlled vivarium.

3. Food and water are available ad libitum in the home cages throughout the experiment.

4. Rats are allowed to acclimatize to the vivarium and housing conditions for one week prior to surgery. During this time rats are left undisturbed with the exception of routine cleaning, feeding, and monitoring of weight.

\section{Intravenous Catheterization Surgery}

1. Under isoflurane gas anesthesia, and using standard aseptic surgical techniques, rats are prepared with silastic catheters in the right jugular vein. The catheter exits into a modified cannula (Plastics One) at the surface of the skull, and the cannula is mounted to the skull with jewelers' screws and dental cement. For details of the surgical procedures, see ${ }^{7,8}$. 
2. At the end of the surgery, catheters are flushed with approximately $0.2 \mathrm{~mL}$ of a solution containing $50 \%$ heparin ( $1000 \mathrm{IU})$ and $50 \%$ dextrose $(25 \mathrm{~g} / 50 \mathrm{~mL})$. A plastic blocker is placed over the opening of the cannula to protect the catheter from external debris, and to maintain catheter patency.

3. At the time of surgery, and again on the day after surgery, rats are administered $5 \mathrm{mg} / \mathrm{kg}$, SC, of the analgesic Ketoprofen.

4. Rats are allowed at least seven days to recover from surgery before experimentation. During this time, rats are left undisturbed in the home cages, with the exception of routine cleaning and feeding, and daily monitoring of weight and general health. On the last day of the recovery period, catheters are flushed with the heparin/dextrose solution (Section 2.2) to verify catheter patency.

\section{Behavioral Procedures}

All behavioral procedures are carried out using equipment supplied by Med Associates Inc. (St Albans, VT). The equipment, housed in a dedicated room, includes a set of standard operant chambers, each contained within a sound-attenuating enclosure. Each operant chamber is equipped with two retractable levers, both elevated $6.5 \mathrm{~cm}$ above a stainless steel rod floor. A white stimulus light is located just above one lever, and responses on that lever (the so-called "active" lever) result in the simultaneous illumination of the stimulus light and activation of an infusion pump (Razel Scientific Instruments, St. Albans, VT). The pump is situated on a shelf just outside the operant chamber, but within the soundattenuating enclosure. The other lever (the so-called "inactive" lever) is left extended in the chamber throughout all experimental procedures; responding on this lever is without consequence. Finally, each chamber is equipped to deliver constant-current, intermittent, inescapable, electric footshock through a scrambler to the steel rod floor. All experimental conditions (e.g., stimulus presentations, drug infusions, shock delivery), as well as data recording and consolidation, are accomplished via interfaces between the test chambers and a central computer operating Med Associates software (MED-IV).

The experiment is carried out in 5 phases: 1) Habituation; 2) Cocaine SA; 3) Drug-free period; 4) Extinction, and 5) Testing for reinstatement. In all phases (except for the drug-free period), rats are transferred for daily sessions from the housing room to the operant test chambers. Animals are transferred individually, in small plastic containers. Food and water are not available in the operant chambers.

1. Habituation

1. Twenty-four hours preceding the first cocaine SA session (see Section 3.2.), rats are allowed to habituate to the operant chambers during a single 2-h session; during this session the active lever is retracted.

2. Cocaine SA training

1. Before each daily training session, rats are weighed in the housing room. Subsequently they are transferred to the operant chambers.

2. Upon being placed in the operant chambers, a spring and tubing assembly (extending from the drug infusion pump) is connected to the skull-mounted cannula.

3. Rats are allowed to self-administer cocaine $\mathrm{HCl}$ during daily 3-h sessions, for a total of 8-10 days (either consecutively or with two intervening "days off" between sessions 5 and 6 ).

4. SA sessions alternate between the morning (9:00-12:00) and afternoon (13:00-16:00). The timing of SA sessions is alternated because the procedures in subsequent phases of the experiment (i.e. extinction and testing) occur at both times of day for all animals (see Sections 3.4 and 3.5); thus, this arrangement prevents any confounding effects of the time of day on subsequent drug-seeking behavior.

5. Each training session is preceded by a 5-min acclimatization period, during which animals are placed in the operant chambers with the active lever retracted.

6. At the beginning of the training session, the active lever is extended into the chamber and the stimulus light (located above the active lever) is illuminated for $30 \mathrm{~s}$. In addition, the house light is activated at this time, and remains illuminated throughout the session. Together, these events signal the availability of cocaine.

7. During the session, responses on the active lever are reinforced by a 3-s infusion of cocaine $(0.23 \mathrm{mg} / 65 \mu \mathrm{L}$, i.v. $)$ on a fixed-ratio- 1 schedule of reinforcement. Concurrent with activation of the infusion pump and drug delivery is a 20 -s activation of the stimulus light above the active lever. This 20 -s light presentation corresponds to a "time-out" period during which additional active lever responses are recorded but not reinforced.

8. Animals are allowed a maximum of 50 infusions per 3-h session. We routinely impose this criterion in our work and, in our experience, have found that it greatly reduces, if not eliminates, incidents of deaths due to overdose.

9. At the end of each daily session, rats' catheters are flushed with $0.2-0.3 \mathrm{~mL}$ of heparin-dextrose solution (see Section 2.2 ) and the rats are returned to their home cage.

3. Drug-free period

1. After the final cocaine SA session, rats are left undisturbed (with the exception of routine cleaning, feeding, and monitoring of weight) in their home cage for a minimum of seven days.

4. Extinction of cocaine-trained behavior

1. Rats are transferred to the operant chambers between 09:00-10:00 for extinction training.

2. The spring and tubing assembly is connected to the skull-mounted cannula.

3. Consistent with the conditions for SA training, each day of extinction training is preceded by a 5-min acclimatization period, during which animals are placed in the operant chambers with the active lever retracted.

4. Rats are then given four 60 -min extinction sessions, separated by 30 -min intervals. Each 60 -min session is initiated by the same events that occur at the start of training sessions (i.e., illumination of the house light, extension of the active lever, and 30-sec illumination of the stimulus light above the active lever). Throughout the sessions, all conditions that were present during SA training are maintained (i.e., response-contingent illumination of stimulus light for $20 \mathrm{sec}$ and 3-s activation of the infusion pump), except that active lever responses are no longer reinforced with cocaine infusions. During the 30-min intervals that separate each 60 -min extinction session, the active lever is retracted and the house light is extinguished.

5. At the end of the last 60-min session, rats are returned to their home cage. 
6. Steps 3.4.1. to 3.4.5. are repeated for 3 consecutive days.

5. Reinstatement of cocaine-trained behavior by footshock stress

1. Rats are transferred to the operant chambers between 09:00-10:00

2. Upon being placed in the operant chambers, the spring and tubing assembly is connected to the skull-mounted cannula.

3. Consistent with the conditions for training and extinction, a 5-min acclimatization period is provided, during which rats are placed in the operant chambers with the active lever retracted.

4. Rats are then given two 60-min extinction sessions (see 3.4.4.) separated by a 30-min interval, identical to those given during the extinction phase.

5. If after the second 60-min session the total number of responses on the previously active lever is less than 15 , animals are given a test for reinstatement after an additional 30-min interval. In this 30-min interval, footshock or sham footshock (i.e., no footshock) is administered (see 3.5.6.). If a criterion of 15 or fewer responses on the active lever is not achieved after the second session, an additional extinction session(s) is given prior to testing for reinstatement.

6. Ten minutes after the second 60-min extinction session (or after any additional 60-min extinction session(s) required to establish the extinction criterion of 15 or fewer responses), animals are exposed to $20 \mathrm{~min}$ of intermittent electric footshock stress (or sham footshock) in the operant chamber. Footshock is given during a time when the active lever is retracted and house and stimulus lights are extinguished. Footshock is delivered according to a variable time schedule at a mean interval of $40 \mathrm{~s}$ (10-70 s range). Each shock is delivered at an intensity of $0.9 \mathrm{~mA}$, and is $0.5 \mathrm{~s}$ in duration. These parameters of footshock are based on extensive pilot work carried out in our lab to establish reliable reinstatement of cocaine seeking, with the minimum intensity and duration of footshock exposure. Under these parameters, animals exhibit a reflexive jumping response to the $0.5 \mathrm{~s}$ shock, and occasionally emit a brief vocalization. Although animals are observed throughout the shock period, the intensity of shock is not of sufficient magnitude to induce more pronounced signs of distress.

7. Immediately following termination of the footshock session, the active lever is extended and rats are allowed to lever press under extinction conditions (i.e., responses are not reinforced with cocaine, but do continue to result in illumination of the stimulus light and activation of the infusion pump). This period of time is defined as the test for reinstatement and is typically 1-3 hours in duration. Most responding occurs in the early part of the test session (first $30 \mathrm{~min}-1 \mathrm{~h}$ ), and subsequently extinguishes over more extended test periods. Thus, a $1 \mathrm{~h}$ test is sufficient to capture the reinstatement effect, with longer test periods providing a more complete extinction profile of the effect.

8. When a repeated measures design is employed, each rat is tested under footshock and sham footshock conditions. Steps 3.5.1. to 3.5.8 are repeated on a subsequent test day, and the alternate test for reinstatement (footshock or sham footshock) is administered.

\section{Representative Results:}
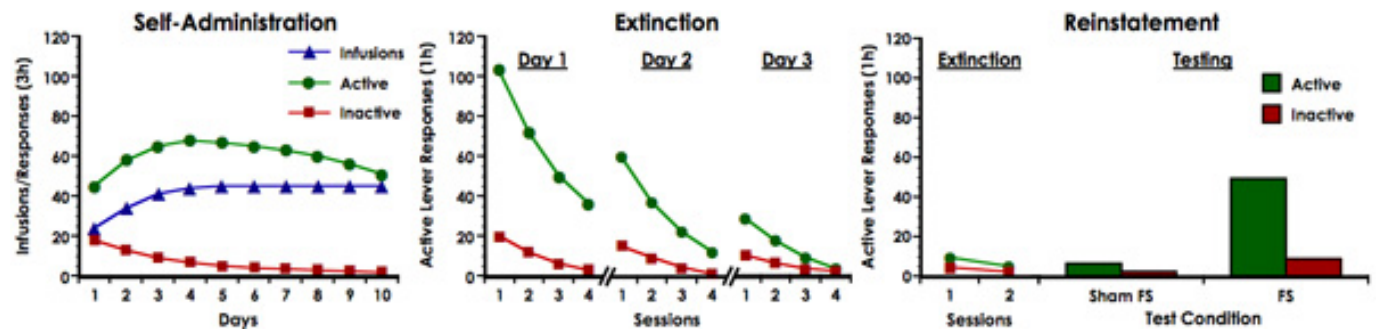

Figure 1. Stylized response profiles for activity on the active and inactive levers during self-administration training, extinction, and reinstatement testing.

Figure 1 presents stylized operant response profiles, typical of those obtained during the various phases of the experimental protocol described herein.

\section{Active lever responses}

1. Cocaine SA training. Figure 1 (left panel) shows a typical cocaine SA training curve that includes both number of infusions and total number of active lever presses. Rats generally acquire cocaine SA within 1-3 sessions and, once the behavior is established, maintain a stable rate of drug intake (typically less than $20 \%$ between-session variance) over subsequent sessions.

2. Extinction of cocaine-trained behavior. It can be seen in Figure 1 (middle panel) that on Day 1 of Extinction, when responses are non-reinforced for the first time, total number of responses on the active lever is initially higher than at the end of the training phase (corresponding to a so-called "extinction burst"); gradually, over the four extinction sessions, responding decreases. On Days 2 and 3 of extinction, some recovery of responding (relative to that observed during the last session of the previous day) may be observed; however, responses are generally fewer than at the start of the previous day of extinction, and extinction generally proceeds more rapidly over the four sessions for that day.

3. Reinstatement of cocaine seeking. It can be seen in Figure 1 (right panel) that 20 min of exposure to intermittent footshock induces an increase in non-reinforced responding on the active lever, relative to that observed at the end of the preceding extinction session. Footshock also induces an increase in responding relative to that observed under conditions in which sham footshock is administered. Thus, the reinstatement of cocaine seeking by footshock stress can be expressed either as: 1) the increased number of non-reinforced responses after exposure to footshock, relative to responses in the extinction session before footshock or 2) the increased number of non-reinforced responses after footshock relative to sham footshock. 


\section{Inactive lever responses}

It can be seen in Figure 1 (red line) that, throughout all experimental phases, there are very low response levels associated with the inactive lever. However, a modest increase in responding on this lever is sometimes observed following exposure to footshock stress. Even in cases in which such an increase in responding occurs, the increase in active lever responding tends to be proportionately much greater.

\section{Discussion}

Over the past few decades, three variations of the SA reinstatement procedure have been developed to study reinstatement of drug seeking in laboratory rats and monkeys. The differences that define the procedural variations relate primarily to whether drug SA, extinction, and testing sessions occur within the same day or on different days ${ }^{1}$. The procedure we have described in this paper is commonly referred to as the "withinbetween" session variation, because drug SA occurs during daily sessions (i.e., in a between-session manner) that are separate from extinction and test sessions, but extinction and testing occur on the same day (i.e., in a within-session manner) [e.g., ${ }^{7,}{ }^{9}$ ]. Alternatively, in so-called "between sessions" procedures, all phases of the experiment occur sequentially, such that SA, extinction, and testing sessions are given on different days (i.e., all phases are between sessions) [e.g., ${ }^{8,10,11}$ ]. In contrast, in complete "within sessions" procedures, SA training, extinction, and test sessions all occur within the same day [e.g., $\left.{ }^{12}\right]$.

Although there are published reports of footshock-induced reinstatement of drug seeking using different variations of the reinstatement procedure, we have found this stressor (at least in animals with a history of cocaine SA) to be most effective when extinction and testing occur on the same day (i.e., in a "within-between" sessions manner). Alternatively, we have had success with obtaining reliable footshock-induced reinstatement of cocaine seeking using a completely "between sessions" procedure, but only under conditions in which animals are housed in the SA chambers throughout the experiment ${ }^{8}$, or at least during extinction and testing ${ }^{13}$. The disadvantage of this between-session approach, over that described in the present protocol, is that it limits the number of subjects that can be trained and tested at one time and, thereby, decreases the efficiency of data collection. Although under the conditions of the present protocol only one group of animals can be extinguished and tested at a given time, a greater throughput can be achieved by staggering by one week the start time of the SA training phase for two groups of rats. In this scenario, a first group of rats can enter the drug-free phase as the second group enters Week 2 of SA training, and, subsequently, extinction and testing can be carried out in the first group while the second group is in its drug-free phase. This approach permits a more efficient use of resources and accelerates the rate of data acquisition, while leading to very effective and reliable footshock-induced reinstatement.

Importantly, in our procedure, extinction of the previously drug-reinforced behavior and testing for reinstatement occurs in the presence of drug-associated cues. Although we have never carried out extinction training or testing in the absence of drug-associated cues, it is our understanding, based on personal communications, that the stressor may in fact be ineffective in inducing reinstatement when testing occurs in the absence of extinguished cues (at least in the case of animals with a history of cocaine SA). A systematic examination of this variable, and the theoretical implications it poses, is an important question for future research.

A final procedural factor that warrants comment is that the effect of footshock stress on reinstatement appears to be highly sensitive to manipulations of context. For example, footshock stress is only effective in inducing the reinstatement of drug seeking if it is administered in the environment in which drug SA and extinction occur; that is, footshock does not reinstate drug seeking if exposure to the stressor occurs in a novel context, suggesting an important interaction between the stressor and drug environment in its effects on reinstatement ${ }^{14}$.

In conclusion, footshock reinstatement procedures, like those described in this paper, have been used with considerable success to elucidate the basic behavioral and neurobiological mechanisms governing the relationship between stress and drug relapse. Indeed, these procedures, when modified to accommodate pharmacological pretreatments or neurochemical manipulations targeting specific neuronal substrates, can serve as powerful tools for characterizing the neurobiology of reinstatement to drug seeking by stress.

\section{Disclosures}

Experiments on animals were performed in accordance with the guidelines and regulations set forth by the Canadian Council of Animal Care and University of Toronto animal care committee. The production of this video was sponsored by Med Associates, Inc. which produces the instrument used in this article.

\section{References}

1. Shaham, Y., et al., The reinstatement model of drug relapse: history, methodology and major findings. Psychopharmacology, 168(1-2): p. 3-20 (2003).

2. de Wit, H., Priming effects with drugs and other reinforcers. Experimental and Clinical Psychopharmacology, 4: p. 5-10 (1996).

3. Bossert, J.M., et al., Neurobiology of relapse to heroin and cocaine seeking: An update and clinical implications. Eur J Pharmacol, 526(1-3): p. 36-50 (2005).

4. Shalev, U., Grimm, J.W., and Shaham, Y. Neurobiology of relapse to heroin and cocaine seeking: a review. Pharmacol Rev, 54(1): p. 1-42 (2002).

5. Shaham, Y. and J. Stewart, Stress reinstates heroin-seeking in drug-free animals: an effect mimicking heroin, not withdrawal. Psychopharmacology, . 119: p. 334-341 (1995).

6. Shaham, Y., Erb, S., and Stewart, J. Stress-induced relapse to heroin and cocaine seeking in rats: a review. Brain Res Brain Res Rev, 33(1): p. 13-33 (2000).

7. Erb, S., et al., Central injections of CRF reinstate cocaine seeking in rats after postinjection delays of up to $3 \mathrm{~h}$ : an influence of time and environmental context. Psychopharmacology (Berl), 187(1): p. 112-20 (2006). 
8. Erb, S., Shaham, Y., and Stewart, J. Stress reinstates cocaine-seeking behavior after prolonged extinction and a drug-free period. Psychopharmacology, 128: p. 408-412 (1996).

9. Tran-Nguyen, L., et al., Time-dependent changes in cocaine-seeking behavior and extracellular dopamine levels in the amygdala during cocaine withdrawal. Neuropsychopharmacology, 19: p. 48-59 (1998).

10. Stretch, R., Gerber, G.J., and Wood, S.M. Factors affecting behavior maintained by response-contingent intravenous infusions of amphetamine in squirrel monkeys. Canadian Journal of Physiology and Pharmacology, 49: p. 581-589 (1971).

11. Shaham, Y., Rodaros, D., and Stewart, J. Reinstatement of heroin-reinforced behavior following long-term extinction Implications for the treatment of relapse to drug-taking. Behavioural Pharmacology, 5: p. 360-364 (1994).

12. de Wit, H. and Stewart, J. Reinstatement of cocaine-reinforced responding in the rat. Psychopharmacology, 75: p. 134-143 (1981).

13. Erb, S., et al., Alpha-2 adrenergic receptor agonists block stress-induced reinstatement of cocaine seeking. Neuropsychopharmacology, 23(2): p. 138-50 (2000).

14. Shalev, U., et al., Stress and relapse to drug seeking in rats: studies on the generality of the effect. Psychopharmacology (Berl), 150(3): $p$. 337-46 (2000). 